\title{
苯的凯库勒式与苯的共振结构
}

杨占会*, 沈旭凡

北京化工大学, 化学学院, 有机化学系, 北京 100029

摘要: 国内外许多有机化学教材认为苯的两个共振极限式是凯库勒式。本文通过梳理苯环结构的研究历史和凯库勒式的 结构特征, 认为该说法并不妥当, 并建议将苯的两个共振极限式命名为 “Pauling 结构式” 或 “Pauling 苯”。

关键词: 有机化学; 苯; 共振结构; 凯库勒式; Pauling 苯

中图分类号：G64；O64；G41

\section{Kekule's Structure of Benzene and the Resonance Structures of Benzene}

\section{Zhanhui Yang ", Xufan Shen}

Department of Organic Chemistry, College of Chemistry, Beijing University of Chemical Technology, Beijing 100029, China.

Abstract: A variety of domestic and international organic chemistry textbooks hold the standpoint that the two resonance structures of benzene are Kekulé structures. By introducing the research history of benzene structures and the structural characteristics of Kekulé's benzene, we propose that the above standpoint should be reconsidered. We also suggest that the two resonance structures should be named as "Pauling's structure" or "Pauling benzene".

Key Words: $\quad$ Organic chemistry; Benzene; Resonance structure; Kekulé's structure; Pauling benzene

苯的共振结构是有机化学教学的基础内容。现行的主流观点认为, 苯环共振于两个极限式i和ii 之间(图1a)。对i和ii结构式的表述, 国内外各大教材说法并不统一。这些教材大体可以分为三派。有 的教材认为, 苯的两个共振极限结构就是凯库勒式 ${ }^{[1-15]}$; 有的教材认为, 苯的两个共振极限式并非 “凯库勒式” ${ }^{[16-23]}$; 还有些教材持保留意见, 回避这一问题 ${ }^{[24-27]}$ 。本文通过介绍苯环结构的研究历 史和分析凯库勒苯的结构特征, 支持 “苯的共振极限式不是凯库勒式”这一观点。

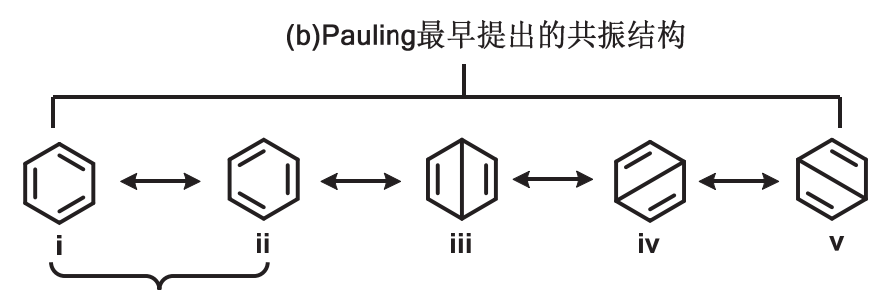

(a)修正后的共振结构

图1 苯的共振结构式

收稿: 2020-09-03; 录用: 2020-09-21; 网络发表：2020-10-09

“通讯作者, Email: zhyang@mail.buct.edu.cn

基金资助: 北京化工大学有机化学优秀本科育人团队项目 
人们对苯的研究已经有近200年的历史。1825年, 英国物理学家、化学家法拉第 ${ }^{[28]}$ 最先从生产照 明气的工业废料中提取出一个新化合物, 并将其命名为 “氢的重碳化合物” (bicarburet of hydrogen)。 1833年, Mitscherlich ${ }^{[29]}$ 在蒸馏安息香胶(含苯甲酸)与生石灰的混合物时, 制备出同样的化合物, 并 将其命名为 “苯”。此后, 人们对苯的结构一直争论不休。需要特别指出的是, 苯的环状结构最早由 Loschmidt ${ }^{[30]}$ 在1861年提出。从1865年开始, 凯库勒陆续提出苯的多个环状结构(图1) ${ }^{[31]}$, 对有机化 学结构理论的发展起到了巨大的 “催化” 作用。如今, 虽然有许多教科书将苯的凯库勒式等同于单 双键交替的正六边形结构, 但历史上凯库勒所提出的苯的 “环状结构” 却并非如此(图2)。1865年,

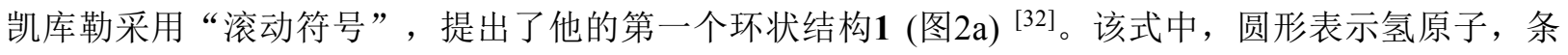
形表示碳原子, 箭头表示碳原子的化学价相互饱和, 并使整体结构呈环状。然而, 该式却不能表明 环中碳原子的实际排列。在 1865 年的另一篇论文中, 凯库勒对上述结构进行了进一步完善, 提出了 六边形结构式 2 (图2b) ${ }^{[33]}$ 。该结构基于三个假设: 苯中的六个碳原子形成一个封闭链; 分子是对称 的; 每个氢原子都与一个碳原子结合。因此, 他认为苯仅存在一种单取代的衍生物和三种双取代衍 生物(邻、间、对)。虽然凯库勒也提出了另一种可能的三角形结构 3 (图 $2 b$ ), 但他却更喜欢六边形结

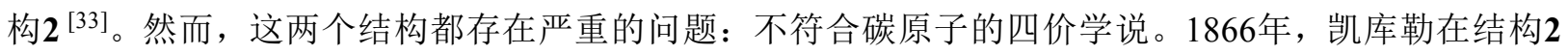
的基础上进行了改进, 提出了三维结构 4 (图2c) ${ }^{[34]}$ 。该结构中, 六个碳原子通过相邻配对的形式, 实现了碳原子的四价, 也满足了碳原子的四面体构型。同时, 他又提出了更加简洁的六边形结构 $\mathbf{5}$ (图 $2 \mathrm{c}$, 来表示结构 4 中的单双键交替情况。虽然结构 5 与我们现在所使用的的苯环结构式几乎相同, 但 它只是结构 $\mathbf{4}$ 的一个简化, 其内涵与今天大不相同。凯库勒认为, 没有一个平面结构式(所有原子都 在一个平面)可以完美表达苯分子中的碳原子连接方式 ${ }^{[35]}$ 。显然, 结构 $\mathbf{5}$ 也不是平面结构, 这可以从 其球棍模型6 中看出(图2d) ${ }^{[18]}$ 。在现代结构理论看来, 凯库勒所提出的苯的结构实际上是一个环己三 烯 (7, 图2e)。虽然凯库勒在1872年为解释邻二取代苯只有一个异构体的现象, 对他的苯环理论进行 了修改, 提出了 “摆动双键学说” (图2f) ${ }^{[36]}$, 但这并不改变结构式5的本质涵义。

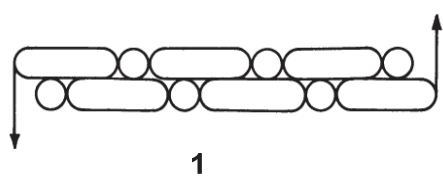

(a)1865年提出

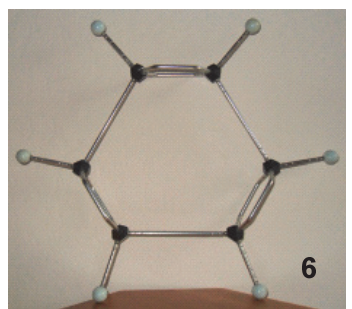

(d)凯库勒结构式的球棍模型

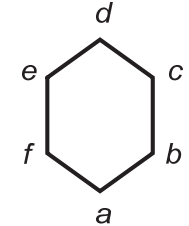

(b) 1865 年提出

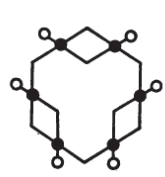

4

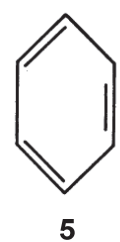

(c)1866年提出

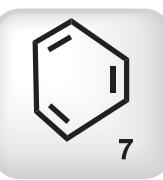

(e)现代结构理论处理后 的凯库勒结构式
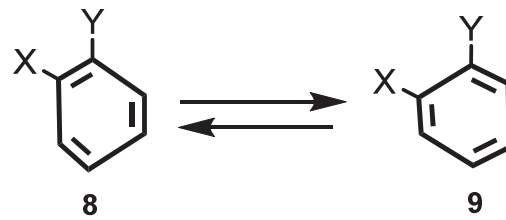

(f) 1872年提出的"摆动双键学说"

\section{图2 凯库勒提出的几种苯的共振结构}

历史上, 六边形结构式 5 并未受到凯库勒的足够重视。在苯的结构理论发表后, 凯库勒撰写了约 30 篇有关芳香物质的论文, 却只在其中的4篇论文中使用过结构式 ${ }^{[37]}$ 。然而, 由于结构式 5 简单明 了, 并且可以用来解释涉及苯环反应的许多问题, 后世学者开始在芳香化合物研究中大量使用结构 式5。随着研究的逐渐深入, 科学界不断赋予该结构式新的涵义, 却渐渐忽略了它的本质——非平面 三维结构式4的简写。1929年, Lonsdale ${ }^{[38]}$ 通过单晶衍射证实了苯环具有平面结构和六个完全相同的 
$\mathrm{C}-\mathrm{C}$ 键, 实际上证明了凯库勒提出的苯的结构是错误的。然而, 科学界却据此将 5 所示的结构认定 为平面结构。1933年, Pauling和Wheland ${ }^{[39]}$ 通过理论计算, 得出了苯环共振于两个 “凯库勒结构” 和三个 “杜瓦苯结构” 的结论(图 1b)。该结论经过进一步修正, 便是如今普遍接受的苯的共振式 (图1a)。后人尊重了 Pauling的说法, 继续在苯的共振结构中沿用了 “凯库勒结构式” 的表述。然而, Pauling的共振理论中, i 和ii都是不存在的极限结构式, 为具有平面结构的单双键交替的正六边形, 且 $C-C$ 单键和 $C=C$ 双键的键长完全相等。这与凯库勒式的内涵相抵触。因此, 将 $\mathbf{i}$ 和 $i i$ 当作是凯库勒 式是明显错误的。确切地说, 应该将 $\mathbf{i}$ 和ii命名为 “Pauling结构式” 或 “Pauling苯”。根据共振理论, 两个 “Pauling苯”可以互相共振, 但两个凯库勒苯之间不存在共振关系(图3)。

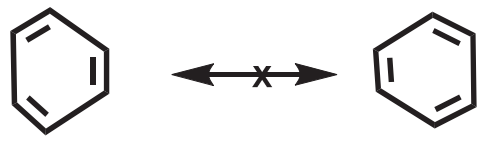

图3 两个凯库勒苯之间不存在共振关系

综上, 本文通过分析苯环结构的研究历史, 比较了凯库勒苯与苯的两个共振极限式的结构特点, 得出了如下结论: 苯的两个共振极限式不是 “凯库勒式”。本文建议将这两个共振极限式命名为

“Pauling结构式” 或 “Pauling苯”。如此, 便有以下建议表述方式: 苯共振于两个Pauling结构式。本 文建议国内外相关有机化学教材能够认真思考本文提出的问题和观点, 重新审视 “苯共振于两个凯 库勒式”的观点。

\section{参 考 文 献}

[1] 邢其毅, 裴伟伟, 徐瑞秋, 裴坚. 基础有机化学. 第4版. 北京: 北京大学出版社, 2016: 712 .

[2] 古练权, 汪波, 黄志纾, 吴云东. 有机化学. 北京: 高等教育出版社, 2008: 91-94.

[3] 鲁崇贤, 杜洪光. 有机化学. 第2版. 北京: 科学出版社, 2009: 8, 198.

[4] 高占先. 有机化学. 第3版. 北京: 高等教育出版社, 2018: 192.

[5] 高占先, 姜文风, 于丽梅. 有机化学简明教程. 北京: 高等教育出版社, 2011: 132.

[6] 常雁红. 有机化学. 北京: 冶金工业出版社, 2016: 80.

[7] 伍越睘, 李伟昶, 沈晓明. 有机化学(修订版). 合肥: 中国科学技术大学出版社, 2002: 170 .

[8] Carey, F. A. Organic Chemistry, 7th ed.; McGraw-Hill Higher Education: New York, USA, 2008; p. 424.

[9] Harold Hart, H.; Hadad, C. M.; Craine, L. E.; Hart, D. J. Organic Chemistry: A Short Course, 13rd ed.; Cengage Learning: Belmont, USA, 2012; p. 117.

[10] Ouellette, R. J.; Rawn, J. D. Principles of Organic Chemistry; Elsevier: Amsterdam, Netherlands, 2015; pp. 134-135.

[11] Wade, L. G., Jr. Organic Chemistry, 8th ed.; Pearson Education, Inc.: Glenview, USA, 2013; p. 714.

[12] Brown, W. H.; Iverson, B. L.; Anslyn, E. V.; Foote, C. S.; Novak, B. M. Organic Chemistry, 8th ed.; Cengage Learning: Boston, USA, $2018 ;$ p. 942.

[13] Carey, F. A.; Giuliano, R. M. Organic Chemistry, 10th ed.; McGraw-Hill Education: New York, USA, 2017; p. 416.

[14] Klein, D. Organic Chemistry, 2nd ed.; John Wiley Sons, Inc.: Hoboken, USA, 2015; p. 838.

[15] Morrison, R. T.; Boyd, R. N. Organic Chemistry, 6th ed.; Prentice-Hall, Inc.: Englewood Cliffs, New Jersey, USA, 1992; p. 503.

[16] Carey, F. A.; Sundberg, R. J. Advanced Organic Chemistry, 5th ed.; Springer: New York, USA, 2005; p. 18.

[17] Solomon, T. W. G.; Fryhel, C. B. Organic Chemistry, 9th ed.; John Wiley \& Sons, Inc.: Hoboken, New Jersey, USA, 2008; p. 603.

[18] Vollhardt, P. K.; Schore, N. Organic Chemistry: Structure and Function, 8th ed.; W. H. Freeman and Company: New York, USA, 2018 ; p. 642.

[19] Smith, J. G. Organic Chemistry, 4th ed.; McGraw-Hill: New York, USA, 2013; p. 632.

[20] 胡宏纹. 有机化学. 第3版. 北京: 高等教育出版社, 2005: 166.

[21] 贾志坚, 武宇芳, 李文娟. 有机化学理论及发展研究. 北京: 中国原子能出版社, 2018: 90. 
[22] Clayden, J.; Greeves, N.; Warren, S. Organic Chemistry, 2nd ed.; Oxford University Press Inc.: New York, USA, 2012 ; p. 144.

[23] 徐寿昌. 有机化学. 第2版. 北京: 高等教育出版社, 2014: 114.

[24] Smith, M. B. March's Advanced Organic Chemistry, 7th ed.; John Wiley \& Sons, Inc.: Hoboken, New Jersey, USA, 2013 ; p. 44.

[25] McMurry, J. Organic Chemistry, 9th ed.; Cengage Learning: Boston, USA, 2016; p. 145.

[26] 覃兆海, 马永强. 有机化学. 北京: 化学工业出版社, 2014: 32.

[27] 李艳梅, 赵圣印, 王兰英. 有机化学. 第2版. 北京: 科学出版社, 2014: 80 .

[28] Faraday, M. Phil. Trans. R. Soc. 1825, 115, 440.

[29] Mitscherlich, E. Ann. Pharm. 1834, 9, 39.

[30] Loschmidt, J. Chemische Studien; Carl Gerold's Sohn: Vienna, Austria-Hungary, 1861; p. 30, 65.

[31] Benfey, O. T. Kekulé Centennial; American Chemical Society: Washington, D. C., USA, 1966; p. 164-166.

[32] Kekulé, F. A. Bull. Soc. Chim. Paris 1865, 3, 98.

[33] Kekulé, F. A. Bull. Acad. Roy. Belg. 1865, 19, 551.

[34] Kekulé, F. A. Liebigs Ann. Chem. Pharm. 1866, 137, 129.

[35] Kekulé, F. A. Zeitschrift für Chem. 1867, 3, 216.

[36] Kekulé, F. A. Liebigs Ann. Chem. Pharm. 1872, 162, 77, 309.

[37] Walker, O. J. Ann. Sci. 1939, 4, 34.

[38] Lonsdale, K. Proc. R. Soc. Lond. 1929, A123, 494.

[39] Pauling, L.; Wheland, G. W. J. Chem. Phys. 1933, 1, 362. 